\title{
Rare B decays with photons in the final state
}

\author{
April 29, 1996
}

Salvatore Mele

Dip. di Scienze Fisiche, Università degli Studi di Napoli "Federico II"

and INFN-Sezione di Napoli, Mostra d'Oltremare, I-80125 Napoli, Italy

\begin{abstract}
A search for rare decays of $B_{d}^{0}$ and $B_{s}^{0}$ mesons with photons in the final state has been performed in more than three million hadronic decays of the $\mathrm{Z}$ collected by the $\mathrm{L} 3$ detector at LEP. Eight exclusive channels have been investigated:

$$
\begin{gathered}
\mathrm{B}_{\mathrm{d}}^{0} \rightarrow \eta \eta, \mathrm{B}_{\mathrm{d}}^{0} \rightarrow \eta \pi^{0}, \mathrm{~B}_{\mathrm{d}}^{0} \rightarrow \pi^{0} \pi^{0}, \mathrm{~B}_{\mathrm{d}}^{0} \rightarrow \gamma \gamma \\
\mathrm{B}_{\mathrm{s}}^{0} \rightarrow \eta \eta, \mathrm{B}_{\mathrm{s}}^{0} \rightarrow \eta \pi^{0}, \mathrm{~B}_{\mathrm{s}}^{0} \rightarrow \pi^{0} \pi^{0}, \mathrm{~B}_{\mathrm{s}}^{0} \rightarrow \gamma \gamma .
\end{gathered}
$$

No candidate has been observed in any of these channels and thus upper limits at $90 \%$ confidence level have been set on the branching ratios. These are the first experimental limits on $\mathrm{B}_{\mathbf{d}}^{0} \rightarrow \gamma \gamma, \mathrm{B}_{\mathbf{d}}^{0} \rightarrow \eta \eta$ and on all the $\mathrm{B}_{\mathbf{s}}^{0}$ modes.
\end{abstract}

Invited talk at the Conference on Production and Decay of Hyperons Charm and Beauty Hadrons, Strasbourg, France, 5-8 Sep. 1995 


\section{Introduction}

Rare B hadron decays are an important test of the Standard Model as well as its extensions and constitute a challenge to detectors' capabilities. High efficiencies and background rejection power are needed to look for a few possible candidates in millions of collected events.

Rare $B$ decays can be searched for at present and future B factories as well as at LEP where $\approx 540,000 \mathrm{~B}_{\mathrm{d}}^{0}$ are available in three million hadronic decays of the $\mathrm{Z}$ collected by the $\mathrm{L} 3$ detector in the years from 1991 through 1994 . Moreover $\approx 160,000 \mathrm{~B}_{\mathrm{s}}^{0}$ are available in the same data sample while they are not accessible at the $\Upsilon(4 \mathrm{~S})$ centre of mass energy. Charge conjugate mesons $\overline{\mathrm{B}}_{\mathbf{d}}^{0}$ and $\overline{\mathrm{B}}_{\mathrm{s}}^{0}$ are also implied throughout this paper.

This paper describes the search for eight rare exclusive decay modes: $\mathrm{B}_{\mathrm{d}, \mathrm{s}}^{0} \rightarrow \eta \eta, \eta \pi^{0}, \pi^{0} \pi^{0}$ and $\mathrm{B}_{\mathrm{d}, \mathrm{s}}^{0} \rightarrow \gamma \gamma$. $\eta$ 's and $\pi^{0}$ 's have been detected by means of their decays into pairs of photons. These searches have been performed with the L3 detector (described in detail in Reference [1]) whose BGO crystal electromagnetic calorimeter has an energy resolution less than $2 \%$ and an angular resolution better than $0.5^{\circ}$ for electromagnetic clusters with energies above $1 \mathrm{GeV}$. Photons are identified using a charged track veto and a study of the consistency of the lateral shower shape with electromagnetic energy deposition as expected from test beam studies.

\section{$2 \quad \mathrm{~B}_{\mathrm{d}, \mathrm{s}}^{0} \rightarrow \eta \eta, \eta \pi^{0}, \pi^{0} \pi^{0}$ decays}

In the Standard Model, the neutral charmless $\mathrm{B}_{\mathrm{d}, \mathrm{s}}^{0}$ decays can occur through a variety of processes such as Cabibbo-suppressed $b \rightarrow u$ transition [2] with a further color suppression with respect to the charged modes $[3]$ or one loop diagrams with a heavy quark and a virtual $\mathrm{W}^{ \pm}$ boson $[4,3]$. Contributions can also arise from electroweak penguins [5].

These decay modes can give information on new physics beyond the Standard Model, since in models with charged Higgs additional diagrams are allowed and can add constructively to the $\mathrm{W}^{ \pm}$boson loop [6]. Minimal Supersymmetric extensions of the Standard Model predict superpartners that could also affect the expected decay rates [7]. A set of diagrams is shown in Reference [4].

The Standard Model theoretical predictions for the branching ratios of the neutral charmless $\mathrm{B}_{\mathrm{d}, \mathrm{s}}^{0}$ decays range from $10^{-5}$ to $10^{-8}[3,9]$. No predictions for extensions to the Standard Model exist. The existing ARGUS and CLEO limits at $90 \%$ confidence level on these decays are $\operatorname{Br}\left(\mathrm{B}_{\mathrm{d}}^{0} \rightarrow \eta \pi^{0}\right)<1.8 \times 10^{-3}[10]$ and $\mathrm{Br}\left(\mathrm{B}_{\mathrm{d}}^{0} \rightarrow \pi^{0} \pi^{0}\right)<9.1 \times 10^{-6}[11]$, respectively.

Since the hard fragmentation of the $b$ quark gives on average $70 \%$ of the beam energy to the $\mathrm{B}_{\mathrm{d}, \mathrm{s}}^{0}$ meson, the $\eta / \pi^{0}$ are likely to have high momentum and the two photons from their decay can have a small opening angle, merging in a single cluster in the electromagnetic calorimeter. This analysis has been performed in the different final state configurations which have shown the best acceptance and background rejection capability:

- $\mathrm{B}_{\mathrm{d}, \mathrm{s}}^{0} \rightarrow \eta \eta$ : four detected photons in the final state or one $\eta$ detected as a photon pair and the other as a single cluster,

- $\mathrm{B}_{\mathrm{d}, \mathrm{s}}^{0} \rightarrow \eta \pi^{0}$ : the $\eta$ detected as a photon pair and the $\pi^{0}$ as a single cluster,

- $\mathrm{B}_{\mathrm{d}, \mathrm{s}}^{0} \rightarrow \pi^{0} \pi^{0}$ : both $\pi^{0}$ 's detected as single clusters.

After the photon and the cluster identification, the global kinematics has been investigated to reject the combinatoric background: 
- The opening angle of the two light mesons is expected to be small, while for random combinations it is peaked toward large values.

- The hard fragmentation of the $b$ quark gives high energy to the $B_{d, s}^{0}$ meson candidate, whereas background tends to be at low energies.

- The cosine of the angle in the $\mathrm{B}_{\mathbf{d}, \mathrm{s}}^{0}$ candidate rest frame between the direction of one decay product and the $\mathrm{B}_{\mathbf{d}, \mathrm{s}}^{0}$ candidate flight direction is peaked for the background, while it is expected to be more isotropic for the signal.

- In decay modes where an $\eta$ is detected as a photon pair, a cut on the invariant mass of these photons can be applied.

- A constrained fit to the $\mathrm{B}_{\mathrm{d}, \mathrm{s}}^{0}$ mass, taking into account the energy and angular resolutions of the calorimeter, has been performed for the $\mathrm{B}_{\mathrm{d}, \mathrm{s}}^{0} \rightarrow \pi^{0} \pi^{0}$ search. The $\chi^{2}$ of this fit shows large values for background and small ones for signal.

The invariant mass of all the photons and/or clusters from the $\mathrm{B}_{\mathbf{d}, \mathbf{s}}^{0}$ candidates, has been calculated for all the decay modes. The resolution was calculated as the width, $\sigma$, of a Gaussian fit to the distribution of this invariant mass for events in signal Monte Carlo surviving the cuts. The invariant mass spectra for data are shown in Figure 1 together with the resolutions.

Since no candidate event has been found in data in a $\pm 2 \sigma$ window around the fit mass of the $\mathrm{B}_{\mathrm{d}, \mathrm{s}}^{0}$ meson, for any of the eight modes, upper limits at $90 \%$ confidence level on the branching ratios have been set and are reported in the upper part of Table 1. Further details on this analysis can be found in in Reference [12].

\section{$3 \quad \mathrm{~B}_{\mathrm{d}, \mathrm{s}}^{0} \rightarrow \gamma \gamma$ decays}

$\mathrm{B}_{\mathrm{d}, \mathrm{s}}^{0} \rightarrow \gamma \gamma$ decays have a clean experimental signature but large theoretical uncertainties affect the few present calculations. These decays can occur via a second order weak transitions with gluonic penguins contributions and are described by diagrams like the ones reported in References [13].

The expected Standard Model value for the branching ratio is of the order of $10^{-7}[14,15]$ and can be significantly enhanced [14] from the existence of charged Higgs bosons expected in extensions to the Standard Model. The search for $\mathrm{B}_{\mathrm{d}, \mathrm{s}}^{0} \rightarrow \gamma \gamma$, like the $\mathrm{b} \rightarrow \mathrm{s} \gamma$ studies, is thus a window on new physics beyond the Standard Model.

Once further calculations on the $\mathrm{B}_{\mathrm{d}, \mathrm{s}}^{0} \rightarrow \gamma \gamma$ processes are performed, the possible observation of $\mathrm{B}_{\mathrm{d}, \mathrm{s}}^{0} \rightarrow \gamma \gamma$ decays could give a measurement of the Cabibbo-Kobayashi-Maskawa matrix elements ratio $\left|\mathrm{V}_{\mathrm{ts}}\right|^{2} /\left|\mathrm{V}_{\mathrm{td}}\right|^{2}$ from the relation:

$$
\frac{\operatorname{Br}\left(\mathrm{B}_{\mathrm{d}}^{0} \rightarrow \gamma \gamma\right)}{\operatorname{Br}\left(\mathrm{B}_{\mathrm{s}}^{0} \rightarrow \gamma \gamma\right)}=\frac{\left|\mathrm{V}_{\mathrm{td}}\right|^{2}}{\left|\mathrm{~V}_{\mathrm{ts}}\right|^{2}} \frac{f_{\mathrm{B}_{\mathrm{d}}^{0} \rightarrow \gamma \gamma}^{2}}{f_{\mathrm{B}_{\mathrm{s}}^{0} \rightarrow \gamma \gamma}^{2}}
$$

This approach is complementary to the study of $\mathrm{B}^{0} \overline{\mathrm{B}}^{0}$ mixing.

A search for $\mathrm{B}_{\mathrm{d}, \mathrm{s}}^{0} \rightarrow \gamma \gamma$ decays has been performed using the L3 detector at LEP [13]. After the identification of photons, the main background is the combinatoric one, which has been reduced with cuts on the $B_{d, s}^{0}$ candidate energy and photons' opening angle. In order to 
separate $\mathrm{b} \overline{\mathrm{b}}$ events from other hadronic events, a multidimensional analysis based on a neural network approach described elsewhere $[16]$ is used.

Figure 2 shows the invariant mass spectra of the two selected photons after the application of all the cuts, for the $\mathrm{B}_{\mathrm{d}, \mathrm{s}}^{0}$ Monte Carlo, the background Monte Carlo and the data sample. The resolutions $\sigma$ are calculated with a Gaussian fit to the $\mathrm{B}_{\mathrm{d}, \mathrm{s}}^{0}$ Monte Carlo invariant mass distribution. No event is observed in a $\pm 2 \sigma$ window around the $\mathrm{B}_{\mathbf{d}, \mathbf{s}}^{0}$ mass and the upper limits at $90 \%$ confidence level on the branching ratios reported in the lower part of Table 1 have been set.

$\mathrm{A} \mathrm{B}_{\mathrm{d}, \mathrm{s}}^{0} \rightarrow \pi^{0} \pi^{0}$ decay can fake a $\mathrm{B}_{\mathrm{d}, \mathrm{s}}^{0} \rightarrow \gamma \gamma$ decay, if the two photons from $\pi^{0}$ decay merge into a single cluster. In calculating the limits on the branching ratio of $\mathrm{B}_{\mathrm{d}, \mathrm{s}}^{0} \rightarrow \gamma \gamma$ the conservative assumption that the contribution from $\mathrm{B}_{\mathrm{d}, \mathrm{s}}^{0} \rightarrow \pi^{0} \pi^{0}$ is negligible has been made and vice-versa. A complementary analysis not relying on the neural network $b \bar{b}$ selection has been performed as a cross-check [17]. This analysis uses photon selection and global kinematic variables similar to those used in the search for $\mathrm{B}_{\mathrm{d}, \mathrm{s}}^{0} \rightarrow \eta \eta, \eta \pi^{0}, \pi^{0} \pi^{0}$ decays. A single variable function based on a multidimensional approach has been used to discriminate the signal from the background events, obtaining compatible results with the analysis described above.

\begin{tabular}{lcccc}
\hline \multicolumn{1}{c}{ Process } & Resolution & Efficiency & $\begin{array}{c}\text { L3 upper limit } \\
90 \% \text { C.L. }\end{array}$ & $\begin{array}{c}\text { Other upper limit } \\
\text { 90\% C.L. }\end{array}$ \\
\hline $\mathrm{B}_{\mathrm{d}}^{0} \rightarrow \eta \eta(\mathrm{I})$ & $107 \pm 10 \mathrm{MeV}$ & $2.5 \pm 0.2_{-0.2}^{+0.2} \%$ & & \\
$\mathrm{~B}_{\mathrm{d}}^{0} \rightarrow \eta \eta(\mathrm{II})$ & $146 \pm 11 \mathrm{MeV}$ & $4.6 \pm 0.3_{-0.2}^{+0.02} \%$ & & - \\
$\mathrm{B}_{\mathrm{d}}^{0} \rightarrow \eta \eta$ & & $4.1 \times 10^{-4}$ & - \\
$\mathrm{B}_{\mathrm{d}}^{0} \rightarrow \eta \pi^{0}$ & $79 \pm 5 \mathrm{MeV}$ & $4.5 \pm 0.3_{-0.03}^{+0.05} \%$ & $2.5 \times 10^{-4}$ & $1.8 \times 10^{-3}$ \\
$\mathrm{~B}_{\mathrm{d}}^{0} \rightarrow \pi^{0} \pi^{0}$ & $97 \pm 4 \mathrm{MeV}$ & $7.6 \pm 0.4_{-0.5}^{+0.2} \%$ & $6.0 \times 10^{-5}$ & $9.1 \times 10^{-6}$ \\
$\mathrm{~B}_{\mathrm{s}}^{0} \rightarrow \eta \eta(\mathrm{I})$ & $101 \pm 10 \mathrm{MeV}$ & $2.4 \pm 0.2_{-0.2}^{+0.2} \%$ & & \\
$\mathrm{~B}_{\mathrm{s}}^{0} \rightarrow \eta \eta(\mathrm{II})$ & $129 \pm 8 \mathrm{MeV}$ & $4.8 \pm 0.3_{-0.3}^{+0.2} \%$ & & - \\
$\mathrm{B}_{\mathrm{s}}^{0} \rightarrow \eta \eta$ & & & $1.5 \times 10^{-3}$ & - \\
$\mathrm{B}_{\mathrm{s}}^{0} \rightarrow \eta \pi^{0}$ & $81 \pm 1 \mathrm{MeV}$ & $4.3 \pm 0.3{ }_{-0.1}^{+0.02} \%$ & $1.0 \times 10^{-3}$ & - \\
$\mathrm{B}_{\mathrm{s}}^{0} \rightarrow \pi \pi^{0}$ & $99 \pm 4 \mathrm{MeV}$ & $8.3 \pm 0.4{ }_{-0.7}^{+0.4} \%$ & $2.1 \times 10^{-4}$ & - \\
\hline $\mathrm{B}_{\mathrm{d}}^{0} \rightarrow \gamma \gamma$ & $73 \pm 3 \mathrm{MeV}$ & $11.8 \pm 0.5 \pm 0.7 \%$ & $3.9 \times 10^{-5}$ & - \\
$\mathrm{B}_{\mathrm{s}}^{0} \rightarrow \gamma \gamma$ & $72 \pm 3 \mathrm{MeV}$ & $11.8 \pm 0.5 \pm 0.7 \%$ & $1.5 \times 10^{-4}$ & \\
\hline
\end{tabular}

Table 1: Resolutions, efficiencies and upper limits for $\mathrm{B}_{\mathrm{d}}^{0}$ and $\mathrm{B}_{\mathrm{s}}^{0}$ branching ratios. The (I) and (II) modes for the $\mathrm{B}_{\mathrm{d}, \mathrm{s}}^{0} \rightarrow \eta \eta$ channel refer respectively to the search for a four photon final state or one with a photon pair and a single cluster. The first error on the efficiencies is statistical, the second systematic.

\section{Conclusions}

A search for rare decays of $\mathrm{B}_{\mathrm{d}}^{0}$ and $\mathrm{B}_{\mathrm{s}}^{0}$ mesons with photons in the final state has been performed in more than three million hadronic decays of the $\mathrm{Z}$ collected by L3 detector at LEP. Eight exclusive modes have been studied:

$$
\mathrm{B}_{\mathrm{d}, \mathrm{s}}^{0} \rightarrow \eta \eta, \mathrm{B}_{\mathrm{d}, \mathrm{s}}^{0} \rightarrow \eta \pi^{0}, \mathrm{~B}_{\mathrm{d}, \mathrm{s}}^{0} \rightarrow \pi^{0} \pi^{0}, \mathrm{~B}_{\mathrm{d}, \mathrm{s}}^{0} \rightarrow \gamma \gamma
$$

No evidence for those decays has been observed and thus upper limits at $90 \%$ confidence level have been set on their branching ratios. These limits, the resolutions and the efficiencies with 
their errors are summarised in Table 1 . The $\mathrm{B}_{\mathrm{d}}^{0} \rightarrow \gamma \gamma, \mathrm{B}_{\mathrm{d}}^{0} \rightarrow \eta \eta$ and all the $\mathrm{B}_{\mathrm{s}}^{0}$ limits are the first ones set while the $\mathrm{B}_{\mathbf{d}}^{0} \rightarrow \eta \pi^{0}$ limit improves the existing one by almost an order of magnitude.

\section{Acknowledgements}

I would like to thank Maneesh Wadhwa for his collaboration.

\section{References}

[1] L3 Collab., B. Adeva et al., Nucl. Instr. Meth. A289 (1990) 35,

O. Adriani et al., Phys. Rep. 236 (1993) 1.

[2] N. Cabibbo, Phys. Rev. Lett. 10 (1963) 531,

M. Kobayashi, T. Maskawa, Prog. Theo. Phys. 49 (1973) 652.

[3] L. L. Chau et al., Phys. Rev. D43 (1991) 2176.

[4] M. Gronau et al., TECHNION-PH-95-10, M. Gronau et al., TECHNION-PH-94-8.

[5] M. Gronau et al., TECHNION-PH-95-11.

[6] S. L. Glashow, E. E. Jenkins, Phys. Lett. B196 (1987) 233.

[7] R. Barbieri, G. F. Giudice, CERN-TH 6830/93.

[8] L3 Collab., M. Acciarri et al., CERN-PPE/95-124.

[9] F. Buccella et al., Il Nuovo Cimento 104 A9 (1991) 1283,

A. Deandrea et al., Phys. Lett. B318 (1993) 549.

[10] ARGUS Collab., H. Albrecht et al., Phys. Lett. B241 (1990) 278.

[11] CLEO Collab., D.M. Asner et al., CLNS 95/1338.

[12] S. Mele, L3 Note \#1817.

[13] L3 Collab., M. Acciarri et al., CERN-PPE/95-136,

T. Aziz et al., L3 Note \#1751,

S. Mele, L3 Note \#1861.

[14] G. Lin et al., Phys. Rev. D42 (1990) 2314.

[15] S. Herrlich, J. Kalinowski, Nucl. Phys. B381 (1992) 501,

J. O. Eeg, I. Picek, Phys. Lett. B336 (1994) 549,

J. Trampetic, FIZIKA B2 (1993) 121.

[16] L3 Collab., O. Adriani et al., Phys. Lett. B307 (1993) 237.

[17] S. Mele, L3 Note \#1873. 


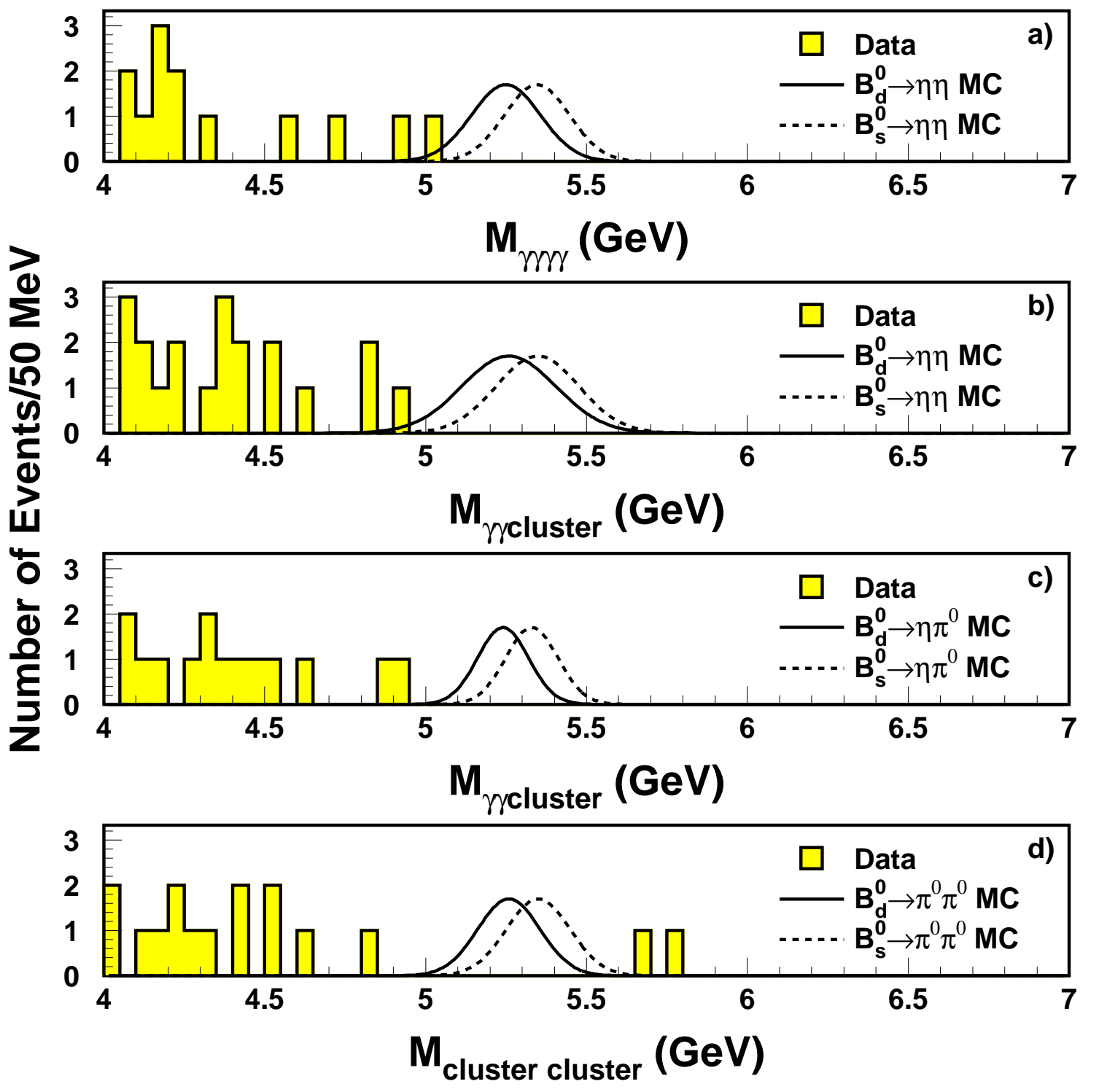

Figure 1: Invariant mass spectra for data and the expected resolutions from the $B_{d, s}^{0}$ Monte Carlo (arbitrary units) after the application of all the cuts. a) $\mathrm{B}_{\mathrm{d}, \mathrm{s}}^{0} \rightarrow \eta \eta$ in four photons, b) $\mathrm{B}_{\mathrm{d}, \mathrm{s}}^{0} \rightarrow \eta \eta$ in a photon pair and one single cluster, c) $\left.\mathrm{B}_{\mathrm{d}, \mathrm{s}}^{0} \rightarrow \eta \pi^{0}, \mathrm{~d}\right) \mathrm{B}_{\mathrm{d}, \mathrm{s}}^{0} \rightarrow \pi^{0} \pi^{0}$ before the application of the $\chi^{2}$ cut. 


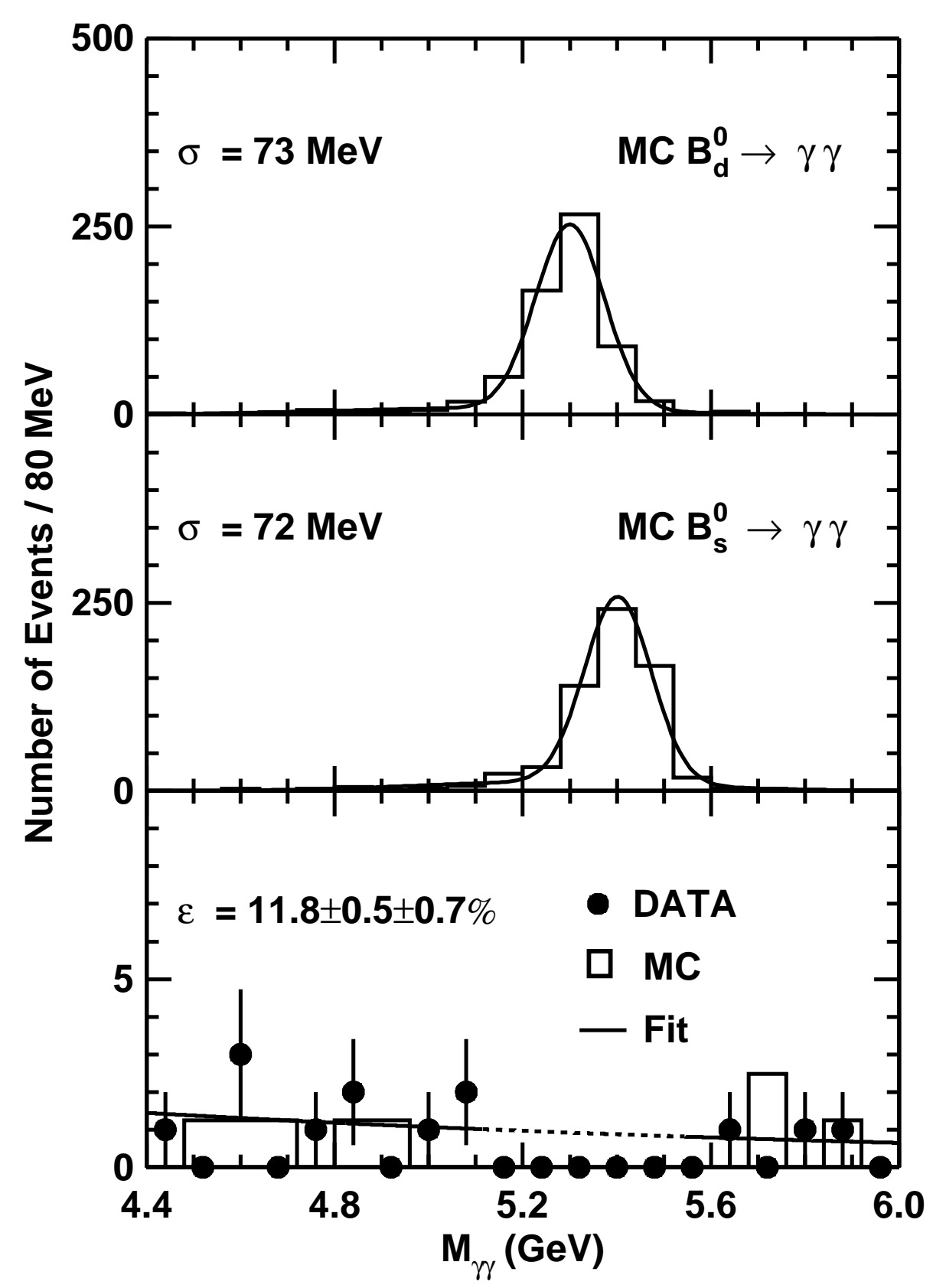

Figure 2: Invariant mass spectra for data and the expected resolutions from the $B_{d}^{0}$ and $B_{s}^{0}$ Monte Carlo. All the cuts are applied. 